\title{
Ansiedade e depressão e uso de substâncias psicoativas em jovens universitários
}

Leopoldo Nelson Fernandes Barbosa ${ }^{1}$

(D) https://orcid.org/0000-0002-0856-8915

Gabriela Catel Abrahamian Asfora ${ }^{1}$

(D) https://orcid.org/0000-0001-6802-4023

Marina Carvalho de Moura ${ }^{1}$

(D) https://orcid.org/0000-0003-1099-5067

Faculdade Pernambucana de Saúde, Psicologia, Recife, PE, Brasil.
Objetivo: identificar a frequência de ansiedade, depressão e uso de substâncias psicoativas em universitários. Método: estudo transversal. Os dados foram coletados utilizando a Escala Hospitalar de Ansiedade e Depressão e questões sobre o uso de substâncias psicoativas. Resultados: foram entrevistados estudantes do primeiro ao sexto período de uma faculdade privada especializada em saúde no nordeste do Brasil. Foi utilizado um questionário sociodemográfico, para caracterizar a população e o uso de substâncias psicoativas, e a Escala Hospitalar de Ansiedade e Depressão. Participaram 116 estudantes e 51,72\% responderam fazer uso de alguma substância psicoativa, principalmente do álcool. Sintomas de ansiedade estiveram presentes em $28,45 \%$ e de depressão em $16,38 \%$. Conclusão: a pesquisa apresentou dados compatíveis com os de outros estudos com esse tipo de população, identificando maior frequência de uso de substâncias em estudantes com sintomas de ansiedade.

Descritores: Abuso de Substâncias; Estudantes; Ansiedade; Depressão.

\section{Como citar este artigo}

Barbosa LNF, Asfora GCA, Moura MC. Anxiety and depression and psychoactive substance abuse in university students. SMAD, Rev Eletrônica Saúde Mental Álcool Drog. 2020;16(1):1-8. doi: https://dx.doi.org/10.11606/issn.1806-6976.smad.2020.155334 


\title{
Anxiety and depression and psychoactive substance abuse in university students
}

\begin{abstract}
Objective: to identify the frequency of anxiety, depression and use of psychoactive substances in university students. Method: cross-sectional study. Data were collected using the anxiety and depression hospital scale and questions on the use of psychoactive substances. Results: students from the first to the six terms of a private college specialized in health care in the northeastern region in Brazil were interviewed. A sociodemographic questionnaire was used to characterize the population and the use of psychoactive substances, in addition to the Hospital Anxiety and Depression Scale. One hundred and sixteen students participated, and $51.72 \%$ reported using some psychoactive substance, especially alcohol. Anxiety symptoms were present in $28.45 \%$ of the participants and depression in $16.38 \%$. Conclusion: the study showed data that were in agreement with those in other studies on this type of population, identifying a higher frequency of substance use by students with anxiety symptoms.
\end{abstract}

Descriptors: Substance Abuse; Students; Anxiety; Depression.

\section{Ansiedad y depresión y abuso de sustancias psicoactivas en estudiantes universitarios}

\begin{abstract}
Objetivo: identificar la frecuencia de ansiedad, depresión y uso de sustancias psicoactivas en universitarios. Método: estudio transversal. Los datos se recopilaron a través de la Escala Hospitalaria de Ansiedad y Depresión y preguntas sobre el uso de sustancias psicoactivas. Resultados: fueron entrevistados estudiantes del primero al sexto período de una facultad privada especializada en salud en el nordeste de Brasil. Se utilizó un cuestionario sociodemográfico para caracterizar la población y el uso de sustancias psicoactivas y la Escala hospitalaria de Ansiedad y Depresión. Participaron 116 estudiantes y el 51,72\% respondió hacer uso de alguna sustancia psicoactiva, principalmente del alcohol. Los síntomas de ansiedad se presentaron en un $28,45 \%$ y la depresión en un 16,38\%. Conclusión: la investigación presentó datos compatibles con otros estudios con ese tipo de población, identificando mayor frecuencia de uso de sustancias en estudiantes con síntomas de ansiedad.
\end{abstract}

Descriptores: Abuso de Sustancias; Estudiantes; Ansiedad; Depresión. 


\section{Introdução}

O uso de drogas ilícitas atinge seu auge entre as idades de 18 e 25 anos. Quase $20 \%$ dessa faixa etária relatam ter usado drogas ilícitas no mês anterior. Quando os jovens adultos se estabelecem, casam e assumem a responsabilidade por seu futuro, eles tendem a interromper o uso de drogas e as taxas de uso caem drasticamente durante a segunda década. Então, continuam a diminuir, embora mais lentamente, à medida que as pessoas entram na vida adulta tardia e velhice ${ }^{(1-2)}$.

A saúde mental de adultos jovens é uma preocupação importante e, entre os diversos transtornos mentais, a depressão é um dos mais comuns nessa etapa da vida. De 15 a $25 \%$ das pessoas podem apresentar crise depressiva pelo menos uma vez na vida, sendo que o primeiro episódio depressivo ocorre, mais frequentemente, antes dos dezoito anos de idade(3). Já os transtornos de ansiedade representam uma das mais comuns e debilitantes formas de psicopatologia na infância e adolescência ${ }^{(4)}$. A depressão é conhecida como o mal do século, um transtorno psíquico de humor que atinge cada vez mais pessoas da sociedade moderna.

A Organização Mundial de Saúde(5) define depressão como um transtorno mental comum, caracterizado não apenas na forma de tristeza como também através de irritabilidade, perda de interesse ou prazer em atividades cotidianas, perda de concentração ou memória, diminuição da autoestima, alteração de sono ou apetite, entre outros sintomas. Esse transtorno pode ter causas genéticas, ambientais e psicossociais e os tratamentos psicoterápicos associados ao uso de medicamentos geram melhores resultados.

A ansiedade é definida como estado de humor desconfortável, apreensão negativa em relação ao futuro e inquietação interna desagradável. Inclui manifestações somáticas e fisiológicas, tais como a sudorese, tremores, tontura e taquicardia, assim como manifestações psíquicas, como inquietação interna, apreensão e desconforto mental(6).

O uso de substâncias psicoativas pode, inicialmente, minimizar ou moderar os sintomas, mas a abstinência e o uso crônico tipicamente os exacerbam em médio prazo. Entende-se por substâncias psicoativas as drogas que são capazes de alterar o funcionamento cerebral, causando modificações no estado mental, no psiquismo e, por isso mesmo, são denominadas drogas psicotrópicas ou substâncias psicoativas. Alguns exemplos desse tipo de drogas são a maconha, cocaína, opioides, tabaco, cafeína, benzodiazepínicos, LSD e anfetaminas, entre outras ${ }^{(7)}$.

Os estudantes universitários são considerados como um grupo de risco para o desenvolvimento de perturbações mentais, como a depressão(8). Os problemas que se podem colocar no período de transição e ao longo da experiência acadêmica, desde mudanças geográficas, rigor acadêmico, novas responsabilidades, afastamento da família, novo ambiente interpessoal, pressão e preocupações com o futuro, dificuldades financeiras, dificuldades de acomodação, entre outros, são situações a que os estudantes têm que se adaptar. No entanto, se muitos estudantes encaram esse novo contexto com alguma naturalidade e como uma experiência positiva, sem experienciar grandes dificuldades de ajustamento, para um número significativo de universitários, todas essas mudanças podem produzir altos níveis de stress que sustentam um conjunto de problemas psicológicos ${ }^{(9)}$.

Assim, esta pesquisa objetivou descrever características psicossociais, identificar a frequência de sintomas de ansiedade e depressão e o uso de substâncias psicoativas em estudantes universitários de um curso de psicologia.

\section{Método}

Trata-se de um estudo quantitativo, de corte transversal, realizado em uma faculdade privada, especializada em saúde, no nordeste do Brasil. A população estudada foi constituída por 116 estudantes que estavam devidamente matriculados no curso de Psicologia e a coleta de dados foi realizada nos meses de abril a junho de 2016. Os dados foram digitados em um banco de dados do Excel e as medias e frequências analisadas no software Epi-Info 7.

A coleta ocorreu no horário em que os estudantes entravam na sala de aula, garantindo que todos eles tivessem a oportunidade de responder ao instrumento utilizado. O protocolo de pesquisa foi entregue para que os estudantes respondessem à pesquisa e foi composto por um questionário estruturado contendo informações sobre características sociodemográficas, dados relacionados ao curso e à utilização de substâncias psicoativas e da Escala Hospitalar de Ansiedade e Depressão $(H A D)^{(10)}$. A HAD é uma escala de rastreio de sintomas constituída por 14 itens, sendo sete destes orientados para avaliação da ansiedade (HAD-A) e os outros sete para a depressão (HAD-D). A escala de medida é de 4 pontos, $0-1-2-3$, podendo atingir 21 pontos cada escala. Zigmond e Snaith ${ }^{(11)}$ recomendam como ponto de corte para ambas as subescalas $\geq 9$ e atribuem: HAD-ansiedade/depressão: sem ansiedade de 0 a 8, com ansiedade $\geq 9$; HAD-ansiedade/depressão entre " 8 " e "10" - "leve", HAD-ansiedade/depressão entre "11 e 14" - moderada HAD- ansiedade/depressão entre "15" e "21" - "grave".

O questionário teve como objetivo descrever o perfil sociodemográfico dos jovens em relação a: sexo, idade, renda e período. Em relação à ansiedade e depressão, determinar a frequência, uso de medicamento, tipo 
de acompanhamento e histórico familiar e também identificar os tipos de substâncias psicoativas utilizadas, a quantidade, o tempo de uso, frequência e motivos de uso. Ao final, analisamos a relação entre ansiedade e depressão e o uso de substâncias psicoativas.

\section{Resultados}

A população estudada foi constituída por 116 alunos que estavam devidamente matriculados no curso de Psicologia, no ano de 2016. A amostra foi calculada no software Epi-Info 6.04.

Foram entrevistados 116 estudantes do curso de psicologia do $1^{\circ}$ ao $6^{\circ}$ períodos de uma faculdade privada. A Tabela 1 apresenta as características sociodemográficas dos estudantes.

Tabela 1 - Características sociodemográficas de estudantes universitários de uma faculdade especializada em saúde. Recife, PE, Brasil, 2019

\begin{tabular}{|c|c|c|}
\hline Variáveis & $\mathbf{N}$ & $\%$ \\
\hline \multicolumn{3}{|l|}{ Quantidade de participantes } \\
\hline Primeiro período & 50 & 43,86 \\
\hline Segundo período & 11 & 9,65 \\
\hline Terceiro período & 4 & 3,51 \\
\hline Quarto período & 17 & 14,91 \\
\hline Quinto período & 23 & 20,18 \\
\hline Sexto período & 9 & 7,89 \\
\hline \multicolumn{3}{|l|}{ Sexo } \\
\hline Feminino & 95 & 82,61 \\
\hline Masculino & 17 & 16,52 \\
\hline Indiferente & 1 & 0,87 \\
\hline \multicolumn{3}{|c|}{ Quantidade de pessoas que residem com os estudantes } \\
\hline De uma a três pessoas & 2 & 1,72 \\
\hline De quatro a sete pessoas & 66 & 56,90 \\
\hline De oito a dez pessoas & 47 & 40,52 \\
\hline Casa própria & 96 & 82,76 \\
\hline Casa alugada & 20 & 17,24 \\
\hline Zona rural & 2 & 1,72 \\
\hline Zona urbana & 113 & 97,41 \\
\hline Comunidade indígena & 1 & 0,86 \\
\hline \multicolumn{3}{|l|}{ Nível de escolaridade do pai } \\
\hline $1^{\mathrm{a}}$ à $4^{\mathrm{a}}$ série do Ensino Fundamental & 7 & 6,09 \\
\hline $5^{\mathrm{a}}$ à $8^{\mathrm{a}}$ série do Ensino Fundamental & 15 & 13,04 \\
\hline Ensino Médio completo & 39 & 33,91 \\
\hline Ensino Superior & 28 & 24,35 \\
\hline Especialização & 19 & 16,52 \\
\hline Não estudaram & 1 & 0,87 \\
\hline Não souberam responder & 6 & 5,22 \\
\hline \multicolumn{3}{|l|}{ Nível de escolaridade da mãe } \\
\hline $1^{\mathrm{a}}$ à $4^{\mathrm{a}}$ série do Ensino Fundamental & 4 & 3,45 \\
\hline $5^{\mathrm{a}}$ à $8^{\mathrm{a}}$ série do Ensino Fundamental & 13 & 11,21 \\
\hline Ensino Médio completo & 37 & 31,90 \\
\hline Ensino Superior & 37 & 31,90 \\
\hline Especialização & 22 & 18,97 \\
\hline Não estudou & 3 & 2,59 \\
\hline \multicolumn{3}{|l|}{ Renda familiar } \\
\hline 10 salários acima & 52 & 45,22 \\
\hline Seis a nove salários mínimos & 23 & 20,00 \\
\hline Três a seis salários mínimos & 19 & 16,52 \\
\hline Um a três salários mínimos & 17 & 14,78 \\
\hline Até um salário mínimo & 4 & 3,48 \\
\hline \multicolumn{3}{|l|}{ Renda Pessoal } \\
\hline Nenhuma renda & 64 & 56,14 \\
\hline Até um salário mínimo & 26 & 22,81 \\
\hline Um a três salários mínimos & 13 & 11,40 \\
\hline Três a seis salários mínimos & 6 & 5,26 \\
\hline Seis a nove salários mínimos & 2 & 1,75 \\
\hline De 10 salários mínimos acima & 3 & 2,64 \\
\hline
\end{tabular}

Participaram da pesquisa $50(43,86 \%)$ estudantes do primeiro período, $11(9,65 \%)$ do segundo, 04 (3,51\%) do terceiro, $17(14,91 \%)$ do quarto, $23(20,18 \%)$ do quinto e $09(7,89 \%)$ do sexto período. Apenas um era estrangeiro. Dos entrevistados, $95(82,61 \%)$ eram do sexo feminino, $17(16,52 \%)$ masculino e $01(0,87 \%)$ respondeu ser indiferente. A idade variou de 17 a 51 anos, com média de 22,9 anos. Dos 114 estudantes que responderam a essa questão, $50(43,86 \%)$ estavam no primeiro período, 11 (9,65\%) no segundo, 04 (3,51\%) no terceiro, $17(14,91 \%)$ no quarto, $23(20,18 \%)$ no quinto e $09(7,89 \%)$ no sexto (Tabela 1$)$.

Sobre a quantidade de pessoas que residiam com os estudantes que responderam ao questionário, 02 $(1,72 \%)$ moravam sozinhos; $66(56,90 \%)$ moravam com uma a três pessoas; $47(40,52 \%)$ afirmaram morar com quatro a sete pessoas e $01(0,86 \%)$ vivia com oito a dez pessoas. Desses, $96(82,76 \%)$ tinham casa própria e $20(17,24 \%)$, alugada; 02 (1,72\%) moravam em zona rural, $113(97,41 \%)$ em zona urbana e $01(0,86 \%)$ em comunidade indígena. Em relação ao nível de escolaridade do pai, 07 (6,09\%) cursaram da $1^{\mathrm{a}}$ à $4^{\mathrm{a}}$ séries do Ensino Fundamental; 15 (13,04\%) da $5^{a}$ à $8^{a}$ série do Ensino Fundamental; 39 (33,91\%) terminaram o Ensino Médio; 28 (24,35\%) completaram o Ensino Superior; 19 (16,52\%) fizeram especialização; $01(0,87 \%)$ não estudou e $06(5,22 \%)$ não souberam responder (Tabela 1 ).

Quanto ao nível de escolaridade da mãe, 04 $(3,45 \%)$ estudaram da $1^{\text {a }}$ à $4^{a}$ série do Ensino Fundamental; $13(11,21 \%)$ da $5^{\text {a à }} 8^{a}$ série do Ensino Fundamental; 37 (31,90\%) cursaram o Ensino Médio; 37 (31,90\%) completaram o Ensino Superior; 22 $(18,97 \%)$ fizeram especialização e 03 (2,59\%) não souberam responder (Tabela 1 ).

Dos 115 estudantes que responderam sobre a renda familiar, a maioria $26(22,61 \%)$ tinha uma renda alta, de mais de 15 salários mínimos, seguida de 23 (20,00\%) com renda de seis a nove salários mínimos; 19 (16,52\%) de três a seis salários mínimos; $17(14,78 \%)$ de um a três salários mínimos. Quinze $(13,04 \%)$ alegaram ter renda aproximada de nove a doze salários mínimos; 11 $(9,57 \%)$ de doze a quinze salários mínimos; 02 (1,74\%) tinham renda de um salário mínimo e 02 (1,74\%) não tinham nenhuma renda. Quanto à renda pessoal, 64 (56,14\%) não possuíam nenhuma renda; 26 (22,81\%) recebiam até um salário mínimo; $13(11,40 \%)$ de um a três salários mínimos; 06 (5,26\%) ganhavam de três a seis salários mínimos; $02(1,75 \%)$ de seis a nove salários mínimos; $01(0,88 \%)$ recebia de nove a doze salários mínimos, a mesma porcentagem de quem respondeu de doze a quinze e mais de quinze salários mínimos (Tabela 1). 
A Tabela 2 apresenta informações sobre o uso de substâncias psicoativas, a frequência e o uso destas por estudantes universitários.

Tabela 2 - Uso de substâncias psicoativas e os principais motivos que levaram ao uso em estudantes universitários de uma faculdade especializada em saúde. Recife, PE, Brasil, 2019

\begin{tabular}{|c|c|c|}
\hline Variáveis & $\mathbf{N}$ & $\%$ \\
\hline \multicolumn{3}{|l|}{ Substâncias Psicoativas } \\
\hline Álcool & 51 & 43,97 \\
\hline Maconha & 21 & 18,26 \\
\hline Nicotina & 11 & 9,48 \\
\hline Ansiolíticos & 11 & 9,48 \\
\hline Antidepressivos & 5 & 4,31 \\
\hline LSD* & 5 & 4,31 \\
\hline Ecstasy & 2 & 1,72 \\
\hline $\mathrm{MDMA}^{\dagger}$ & 2 & 1,72 \\
\hline $\begin{array}{l}\text { Outras substâncias (Loló, cocaína, cola, special } \\
k \text {, cogumelos, rapé) }\end{array}$ & 6 & 5,20 \\
\hline \multicolumn{3}{|l|}{ Frequência de uso } \\
\hline Diário & 11 & 18,64 \\
\hline Dois a três dias por semana & 3 & 5,08 \\
\hline Finais de semana & 20 & 33,90 \\
\hline Mensalmente & 10 & 16,95 \\
\hline Quase nunca & 15 & 25,42 \\
\hline \multicolumn{3}{|l|}{ Tempo de uso } \\
\hline Menos de um ano & 5 & 8,47 \\
\hline Um ano & 3 & 5,08 \\
\hline Dois anos & 7 & 11,86 \\
\hline Três anos & 7 & 11,86 \\
\hline Quatro anos & 5 & 8,47 \\
\hline Cinco anos acima & 32 & 54,20 \\
\hline \multicolumn{3}{|l|}{ Principais motivos de uso } \\
\hline Curiosidade & 8 & 14,29 \\
\hline Diversão & 4 & 7,14 \\
\hline Vontade própria & 3 & 5,36 \\
\hline Socialização & 2 & 3,57 \\
\hline
\end{tabular}

Entre participantes da pesquisa, 60 (51,72\%) responderam fazer uso de alguma substância psicoativa. O uso de álcool prevaleceu, com 51 (43,97\%) das respostas, seguido da maconha com 21 (18,26\%). A nicotina e ansiolíticos apresentaram a mesma porcentagem - 11 (9,48\%). Antidepressivos e LSD também obtiveram o mesmo score - 05 (4,31\%), assim como ecstasy e MDMA - 02 (1,72\%), seguidos de loló $01(0,88 \%)$, cocaína - $01(0,87 \%)$, cola - $01(0,87 \%)$, special $K$ - $01(0,86 \%)$, cogumelos - $01(0,86 \%)$ e rapé - 01 (0,86\%). A frequência de uso foi de 11 (18,64\%) para uso diário e $03(5,08 \%)$ de dois a três dias por semana. Vinte $(33,90 \%)$ alegaram fazer o uso de substâncias somente nos fins de semana, 10 (16,95\%) mensalmente e 15 (25,42\%) quase nunca (Tabela 2$)$.

Quanto ao tempo de uso, $05(8,47 \%)$ alegaram fazer uso de substâncias psicoativas havia menos de um ano; 03 (5,08\%) havia um ano; 07 (11,86\%) havia dois anos, mesmo score atingido por quem fazia o uso havia três anos; 05 (8,47\%) fazia uso havia quatro anos e 32 $(54,20 \%)$ havia cinco anos ou mais. Dentre os motivos de uso, destacaram-se: curiosidade - 08 (14,29\%), diversão - 04 (7,14\%), vontade própria - 03 (5,36\%) e socialização - 02 (3,57\%). Outros motivos citados para justificar o uso foram depressão, busca por relaxamento, prescrição psiquiátrica, ansiedade, experimentar as sensações provocadas no corpo e motivos de humor, fugir da realidade, insatisfações no ambiente familiar, busca de conforto, vazio existencial, entre outros, e atingiram a porcentagem de $1,79 \%$ (o que corresponde a um participante) (Tabela 2). A Tabela 3, a seguir, apresenta informações sobre antecedentes familiares, ansiedade e depressão dos estudantes.

Tabela 3 - Antecedentes familiares, ansiedade e depressão de estudantes universitários de uma faculdade especializada em saúde. Recife, PE, Brasil, 2019

\begin{tabular}{lcc}
\hline \multicolumn{1}{c}{ Variáveis } & N & $\%$ \\
\hline Histórico familiar & & \\
$\quad$ Ansiedade & 13 & 11,30 \\
$\quad$ Depressão & 26 & 22,61 \\
$\quad$ Ambos & 39 & 33,91 \\
Histórico pessoal & & \\
$\quad$ Consideram-se ansiosos & 83 & 71,55 \\
$\quad$ Já foram diagnosticados com depressão & 22 & 18,97 \\
$\quad$ Fizeram ou faziam uso de medicamento para & 33 & 28,45 \\
$\quad$ ansiedade e/ou depressão & & \\
$\quad$ Tiveram ou tinham algum tipo de & 67 & 57,76 \\
$\quad$ acompanhamento psicológico & 33 & 28,45 \\
$\quad$ Apresentavam sintomas de ansiedade (HAD-A) & 19 & 16,38 \\
$\quad$ Apresentavam sintomas depressão (HAD-D) & & \\
*HAD-A = Escala Hospitalar de Ansiedade e Depressão & &
\end{tabular}

Sobre o histórico familiar de ansiedade e/ou depressão, 39 (33,91\%) referiram sim para ambos, 37 $(32,17 \%)$ não, $26(22,61 \%)$ apenas depressão e 13 $(11,30 \%)$. apenas ansiedade. Cerca de $83(71,55 \%)$ estudantes se consideraram ansiosos e, entre eles, 22 (18,97\%) já tinham sido diagnosticados com depressão; $33(28,45 \%)$ já tinham feito ou faziam uso de algum medicamento para depressão e/ou ansiedade. Algum tipo de acompanhamento psicológico foi referido por $67(57,76 \%) ; 33(28,45 \%)$ apresentavam sintomas de ansiedade e $19(16,38 \%)$ apresentavam sintomas depressivos (Tabela 3 ).

\section{Discussão}

Sociologicamente, as pessoas podem ser consideradas adultas quando são responsáveis por si mesmas ou escolhem uma carreira, casam-se ou estabelecem um relacionamento afetivo significativo ou iniciam uma família. A maturidade psicológica, contudo, depende de realizações, como descobrir a própria identidade, tornar-se independente dos pais, desenvolver um sistema de valores e estabelecer relacionamentos ${ }^{(11)}$. Outros autores afirmam; ainda. que a entrada na vida adulta é marcada não apenas por critérios externos, mas por indicadores internos como o sentimento de autonomia, autocontrole, e responsabilidade pessoal, ou 
seja, é mais um estado de espírito do que um evento isolado(11). Assim, a entrada na universidade pode resultar em uma ampla gama de necessidades adaptativas na vida dos estudantes em todo o mundo.

Um estudo realizado na França com 1.743 estudantes do $1^{\circ}$ ano da universidade apontou uma prevalência de mal-estar psicológico, associado com o aumento do risco de ansiedade e depressão, que foi estimada em $15.7 \%$ dos jovens do sexo masculino e em 33\% do sexo feminino(12). Na Turquia, os resultados na população universitária mostram uma situação preocupante. Em termos de depressão, ansiedade e stress, foram encontrados níveis de moderada ou elevada severidade em $27.1 \%, 47.1 \%$ e $27 \%$ dos respondentes, respectivamente ${ }^{(13)}$.

No Brasil, estudantes de medicina da Universidade Federal de Uberlândia, avaliados com o Inventário de Beck, apresentaram uma prevalência de sintomas depressivos de $79 \%$, sendo $29 \%$ em grau leve; $31 \%$ em grau moderado e $19.25 \%$ em grau considerado grave $^{(14)}$. Outra pesquisa, que analisou a sintomatologia de depressão e ansiedade em 200 estudantes de uma universidade privada do Rio Grande do Sul, concluiu que as mulheres apresentaram níveis significativamente mais altos que os homens tanto em ansiedade quanto em depressão. Em relação ao período no curso, os alunos de início de curso apresentaram índices significativamente mais altos de depressão que os de final de curso, não havendo diferenças para os níveis de ansiedade. Alunos dos cursos de Letras e Psicologia apresentaram níveis maiores de depressão que os outros e, em relação à ansiedade, não houve diferença estatisticamente significativa, mas os alunos do curso de Letras também apresentaram médias mais altas ${ }^{(15)}$.

No presente estudo foi constatado que, dos 22 estudantes que responderam já terem sido diagnosticados com depressão, $18(81,81 \%)$ eram mulheres, o que se assemelha ao observado no estudo citado acima. Outro dado que é compatível com os do estudo acima é em relação ao período, pois, de cinquenta estudantes do primeiro período, 17 (34,00\%) apresentavam traços de ansiedade ou depressão, um número considerável, prevalecendo sobre outros períodos.

A depressão atinge, em maior escala, as mulheres. Estudos mostram que esse fato se deve a uma correlação entre o avanço da puberdade e sintomas depressivos. Outros possíveis fatores são o modo como as meninas são socializadas e sua maior vulnerabilidade ao estresse nas relações sociais. Alguns outros fatores de risco para a depressão são ansiedade, medo do contato social, eventos estressantes, doenças crônicas, conflitos entre pais e filhos, abuso ou negligencia, uso de álcool ou drogas e ter um dos pais com histórico de depressão. Esses fatores independem de gênero(1). Foi constatado, pelos estudos dos resultados de pesquisas já realizadas sobre o tema, que a ansiedade também atinge as mulheres em maior escala.

Na revisão de literatura, deparamo-nos com vários artigos que relacionam o abuso de substâncias psicoativas com a ansiedade e depressão, que podem motivar indivíduos a fazerem o uso de drogas para aliviar o próprio mal-estar. Uma pesquisa realizada em Maceió (AL) ${ }^{(16)}$, que relaciona o uso de substâncias psicoativas e a ansiedade, com 407 estudantes de 14 a 18 anos, constatou que $82,10 \%$ afirmaram já ter feito o uso de substâncias psicoativas; $71 \%$ usaram por pelo menos um ano e $31,40 \%$ por um mês; $12,8 \%$ usavam frequentemente e $13,5 \%$ faziam uso pesado. Apenas $17,4 \%$ dos participantes responderam nunca ter consumido nenhum tipo de substância psicoativa. No nosso estudo foi constatado que $60(51,72 \%)$ participantes responderam fazer uso de alguma substância psicoativa.

O uso de substância psicoativa cai drasticamente na segunda década de vida, o que possivelmente explica essa divergência(1). Em relação à ansiedade, os participantes de Maceió(16) apresentaram uma distribuição de 24,8\% para grau mínimo, 23,6\% leve, $28 \%$ moderado e $23,6 \%$ grave. Ao final, concluiu-se que, os que responderam afirmativamente quanto ao uso/frequência de substâncias, como álcool, cigarros, solventes e energéticos, apresentaram maior prevalência de ansiedade moderada. No nosso estudo, $28,45 \%$ apresentaram sintomas de ansiedade verificados pelo ponto de corte da escala, mas chama à atenção a informação autorreferida de que $71,55 \%$ deles se consideravam ansiosos.

As pesquisas têm apontado que os universitários estão inseridos numa população de vulnerabilidade mais propensa a desenvolver transtornos mentais que podem levar ao abuso de substâncias psicoativas. Diante disso, espera-se, por meio dessa pesquisa, poder contribuir para a investigação da possível relação entre ansiedade e depressão e o abuso de substâncias psicoativas por parte dos universitários, e, assim, detectar precocemente e prevenir esse tipo de problema. Como limitações deste estudo, ressaltamos que não foram realizadas análises estatísticas para correlacionar as variáveis e os dados correspondem a um corte transversal.

\section{Conclusão}

Este estudo buscou descrever a frequência de sintomas de ansiedade e depressão e o consumo de substâncias psicoativas em jovens universitários e identificou que a maioria dos estudantes que apresentaram sintomas de ansiedade fazia o uso de alguma substância psicoativa, lícita ou não. Esses dados são compatíveis com os de outras pesquisas realizadas com essa população. 
Entre as substâncias psicoativas citadas pelos estudantes, o álcool era o mais usado, provavelmente, pelo fato de este ser lícito, de fácil acesso, culturalmente utilizado e aceito. Em segundo lugar esteve a maconha, substância que vem ganhando cada vez mais espaço na mídia e em estudos científicos e, por fim, o tabaco ficou em terceiro lugar junto ao uso de ansiolíticos.

A caracterização de aspectos psicossociais, da saúde mental e da frequência de uso de substâncias psicoativas em uma população vulnerável pode servir de importante fonte para o desenvolvimento de futuros programas de prevenção e atenção à saúde dos universitários. Em relação a uso de substâncias psicoativas, ansiedade e depressão, programas psicoeducacionais podem ser uma alternativa importante, pois podem desmistificar o que de fato são sintomas de ansiedade e depressão e assim propiciar suporte adequado.

\section{Referências}

1. Papalia D, Feldman R. Desenvolvimento Humano. 12ed. Porto Alegre: Artmed; 2013.

2. Substance Abuse and Mental Health Services Administration (SAMHSA). Results From 2008 National Survey On Drug Use And Health: National Findings. Rockville; 2009.

3. Bahls S, Bahls F. Depressão na adolescência: características clínicas. Interação em Psicologia, 2002. Disponível em: https://revistas.ufpr.br/psicologia/ article/view/3193. Acesso: 12 ago 2019. doi: http:// dx.doi.org/10.5380/psi.v6i1.3193

4. Vianna RRAB. Avaliação dos níveis de ansiedade de uma amostra de escolares no Rio de Janeiro através da Escala Multidimensional de Ansiedade para Crianças (MASC-VB). Rio de Janeiro; 2009. Disponível em: http:// dx.doi.org/10.1590/S0103-56652009000200029. Acesso: 15 jul 2016.

5. Organização Mundial da Saúde. Classificação de Transtornos Mentais e de Comportamento da CID-10. Descrições Clínicas e Diretrizes Diagnósticas. Porto Alegre: Artes Médicas; 1993.

6. Dalgalarrondo P. Psicopatologia e semiologia dos transtornos mentais. 2.ed Porto Alegre: Artmed; 2008.

7. Da Silva JLB. Estudo das Substâncias Psicoativas. Santa Catarina; 2010. Disponível em: <http:// www.direcionaleducador.com.br/drogas/modulo-ii$\%$ E2\%80\%93-estudo-das-substancias-psicoativas>. Acesso: 6 jul 2016.

8. Vázquez FL, Blanco V. Prevalence of DSM-IV major depression among spanish university students. J Am Coll Health. 2008. Available from: https://doi.org/10.3200/ JACH.57.2.165-172 Acesso: 1 ago 2019.

9. Bouteyre E, Maurel M, Bernaud J. Daily hassles and depressive symptoms among first year psychology students in France: The role of coping and social support. Stress and Health: J Int Soc Invest Stress. 2007;23(2):93-9. Available from: http://dx.doi. org/10.1002/smi.1125 Acesso: 1 ago 2019.

10. Zigmond A, Snaith RP. The Hospital Anxiety and Depression Scale. Acta Psychiatrica Scand. 1983. Available from: https://doi.org/10.1111/j.1600-0447.1983. tb09716.x Acesso em: 1 ago 2019.

11. Shanahan MJ, Porfeli EJ. Mortimer JT, Erickson LD. Subjective Age Identity and the Transition to Adulthood: When Do Adolescents Become Adults? In: Settersten RA Jr, Furstenberg FF, Rumbaut RG, editors. On the Frontier of Adulthood: Theory, Research, and Public Policy. Chicago: University of Chicago Press; 2005.

12. Verger P, Combes JB, Kovess-Masfety V, Choquet M, Guagliardo V, Rouillon F, Peretti-Wattel P. Psychological distress in first year university students: socioeconomic and academic stressors, mastery and social support in young men and women. Soc Psychiatr Epidemiol. 2009;44:643. Available from: https://doi.org/10.1007/ s00127-008-0486-y Acesso: 1 ago 2019.

13. Bayram N, Bilgel N. The prevalence and sociodemographic correlations of depression, anxiety andstressamong a group of university students. Soc Psychiat Epidemiol. 2008;43: 667. Available from: https:// doi.org/10.1007/s00127-008-0345-x Acesso: 1 ago 2019. 14. Abrão $C B$, Coelho EP, Passos LBS. Prevalência de sintomas depressivos entre estudantes de medicina da Universidade Federal de Uberlândia, 2008. Rev Bras Educ Médica. 2008; 32(3):315-23. Disponível em: https:// dx.doi.org/10.1590/S0100-55022008000300006 Acesso: 1 ago 2019.

15. Brandter M, Bardagi M. Sintomatologia de Depressão e Ansiedade em Estudantes de uma Universidade Privada do Rio Grande do Sul. Porto Alegre; 2009. Disponível em: http://www.fafich.ufmg.br/gerais/index.php/gerais/ article/viewFile/67/49. Acesso: 1 ago 2019.

16. Lopes AP, Rezende MM. Ansiedade e consumo de substâncias psicoativas em adolescentes. Est Psicol. (Campinas) 2013;30(1):49-56. Disponível em: https:// dx.doi.org/10.1590/S0103-166X2013000100006 Acesso: 1 ago 2019.

17. Brandtner M, Bardagi M. Sintomatologia de depressão e ansiedade em estudantes de uma universidade privada do Rio Grande do Sul. Gerais: Rev Interinstitucional Psicol. 2009;2(2): 81-91. Disponível em: http://pepsic.bvsalud.org/scielo.php?script=sci_ arttext\&pid=S1983-82202009000200004\&Ing=pt\&tlng =pt. Acesso: 1 ago 2019 . 


\section{Contribuição dos autores}

Concepção e planejamento do estudo: Leopoldo Nelson Fernandes Barbosa. Obtenção dos dados: Gabriela Catel Abrahamian Asfora e Marina Carvalho de Moura. Análise e interpretação dos dados: Leopoldo Nelson Fernandes Barbosa, Gabriela Catel Abrahamian Asfora e Marina Carvalho de Moura. Redação do manuscrito: Gabriela Catel Abrahamian Asfora e Marina Carvalho de Moura. Revisão crítica do manuscrito: Leopoldo Nelson Fernandes Barbosa.

Todos os autores aprovaram a versão final do texto.

Conflito de interesse: Os autores declaram não haver conflito de interesse.

Esta licença permite que outros remixem, adaptem e criem a partir do seu trabalho para fins não comerciais, e embora os novos trabalhos tenham de lhe atribuir o devido crédito e não possam ser usados para fins comerciais, os usuários não têm de licenciar esses trabalhos derivados sob os mesmos termos. 